\title{
Intimate Partner Violence as a Risk Factor For Self- harm Behaviors: A Meta-analysis
}

\author{
Yumeng Chen* \\ College of Arts and Sciences, Emory University, Atlanta, Georgia 30322, USA \\ *Corresponding author. Email: margy.chen@emory.edu
}

\begin{abstract}
Non-suicidal self-injury (NSSI) is a self-damaging behavior which brings substantial harm to the individual. Exposure to intimate partner violence in the past may be a predictor variable that is related to NSSI. However, little is known about the nature or magnitude of this relationship. This meta-analysis therefore aimed to provide a wholistic view of existing literature to capture and quantify this potential relationship between intimate partner violence and NSSI. The databases PubMed, Scopus, and the Web of Science were searched for relevant articles. This yielded 6 studies and 8 independent samples published after 2000. The present random-effects meta-analysis identified that those who had experienced intimate partner violence were significantly more at risk for developing NSSI. This finding targeted specific population at risk for developing NSSI and offered insights about providing necessary intervention for vulnerable individuals.
\end{abstract}

Keywords: intimate partner Violence, non-suicidal self-injury, deliberate self-harm, dating violence

\section{INTRODUCTION}

Non-suicidal self-injury (NSSI) could be characterized as the causing of intential, direct damage to one's own body without aiming to commit suicide[1]. Common methods included scratching, cutting, scraping, or carving one's skin[2]. NSSI has developed into a leading public health issue, while being highly frequent among teenagers and young adults[3]. People who undertook NSSI behaviors comprised a heterogenous group, as multiple potential risk factors could contribute to the development, engagement, and perpetuation of such behaviors. A large body of literature has addressed how self-injurious behaviors were correlated with psychiatric disorders and later suicidal attempts[4,5]. Therefore, knowledge and understanding of the psychological variables associated with NSSI would be beneficial towards both preventing suicides by identifying vulnerable groups and developing specific intervention methods which would suit their circumstances.

It has also been reported that NSSI behaviors were often observed in individuals suffering from posttraumatic stress disorder (PTSD) symptoms and up to $79 \%$ of those suffering have experienced rejection or violence in the past[6]. Although the development of posttraumatic stress disorder (PTSD) was found to be related to various risk factors, being involved in intimate partner violence (IPV) and especially IPV victimization has become more and more prominent[7,8]. Intimate partner violence could be understood as any physical, sexual, or psychological abuse inflicted by a current or former partner. Although IPV could also be directed towards men, women were more vulnerable to suffer from more severe and chronic patterns of violence and control, leading to a greater level of fear and injury[9]. Moreover, cross-sectional studies have found that history of IPV was consistently and strongly correlated with depressive symptoms, depressive disorders, suicidal ideations, and suicidal attempts among women[10-12]. In addition, empirical evidence has shown that engagement in NSSI behaviors could alleviate negative emotions caused by adverse events for some individuals[13]. For instance, domestic violence victimization was a strong predictor for self-destructive behaviors, higher tolerance for bodily harm, and more negative self-image[14]. Given these conditions closely related to NSSI, investigating the role of a psychosocial variable such as IPV history would be useful in interpreting the etiology of NSSI behaviors. 


\subsection{Aim}

Previous research has proposed the possible relationship between exposure to intimate partner violence and the development of non-suicidal self-injury, but literature on this potential association has not been systematically reviewed. Thus, a meta-analysis was conducted to systematically detect and measure the association between intimate partner violence and nonsuicidal self-injury.

\section{METHOD}

\subsection{Inclusion and exclusion criteria}

Only studies informing original research findings on the relationship between a history of intimate partner violence and non-suicidal self-injurious behaviors were included for this review. Studies published before 2000 were not included in order to provide a more recent outlook of research related to the topic. Articles were firstly assessed based on titles and abstracts to determine their relevance for this review. Full-text documents of relevant studies were then obtained and assessed for inclusion in the final review. For determining relevant studies for this review, the following inclusion criteria were used: The article reported original finding, either from primary research or secondary data analysis. The article needed to be empirical and quantitative. The article reported on the relationship between IPV and NSSI. The article had been published between January 2000 and August 2021. The article needed to be available in English.
Studies in which all participants had exposure to intimate partner violence or all participants had histories of non-suicidal self-injurious behaviors were excluded, since such studies could not provide insights about the association between the two variables ${ }^{[10-12]}$. Studies that only examined self-injurious behavior with suicidal intent, or that did not differentiate between participants who had suicidal intent and those who did not, were also excluded $^{[15]}$. Finally, studies that were not published in English were excluded.

\subsection{Search strategy}

Literature searches were undertaken on three online databases (Scopus, PubMed, and the Web of Science), including literature published during the time period of January 2000 to August 2021. This time scope kept the body of reviewed literature to a manageable size while making sure relatively recent publications were obtained. Due to ambiguity regarding terminologies used, multiple keywords were identified. Moreover, the Boolean logic terms "or" and "and" were used to combine searches. After combining all possible keywords, the following search string was used: (self-injurious behavior OR selfmutilation OR deliberate self-harm OR self-mutilative behavior OR self-destructive behavior OR non-suicidal self-injury) AND (intimate partner violence OR domestic violence OR dating violence OR spouse abuse OR partner abuse). For each databse, searches were conducted within the titles, abstracts, and keyword lists. For more information, Figure. 1 outlines how articles were identified, screened, and assessed for inclusion.

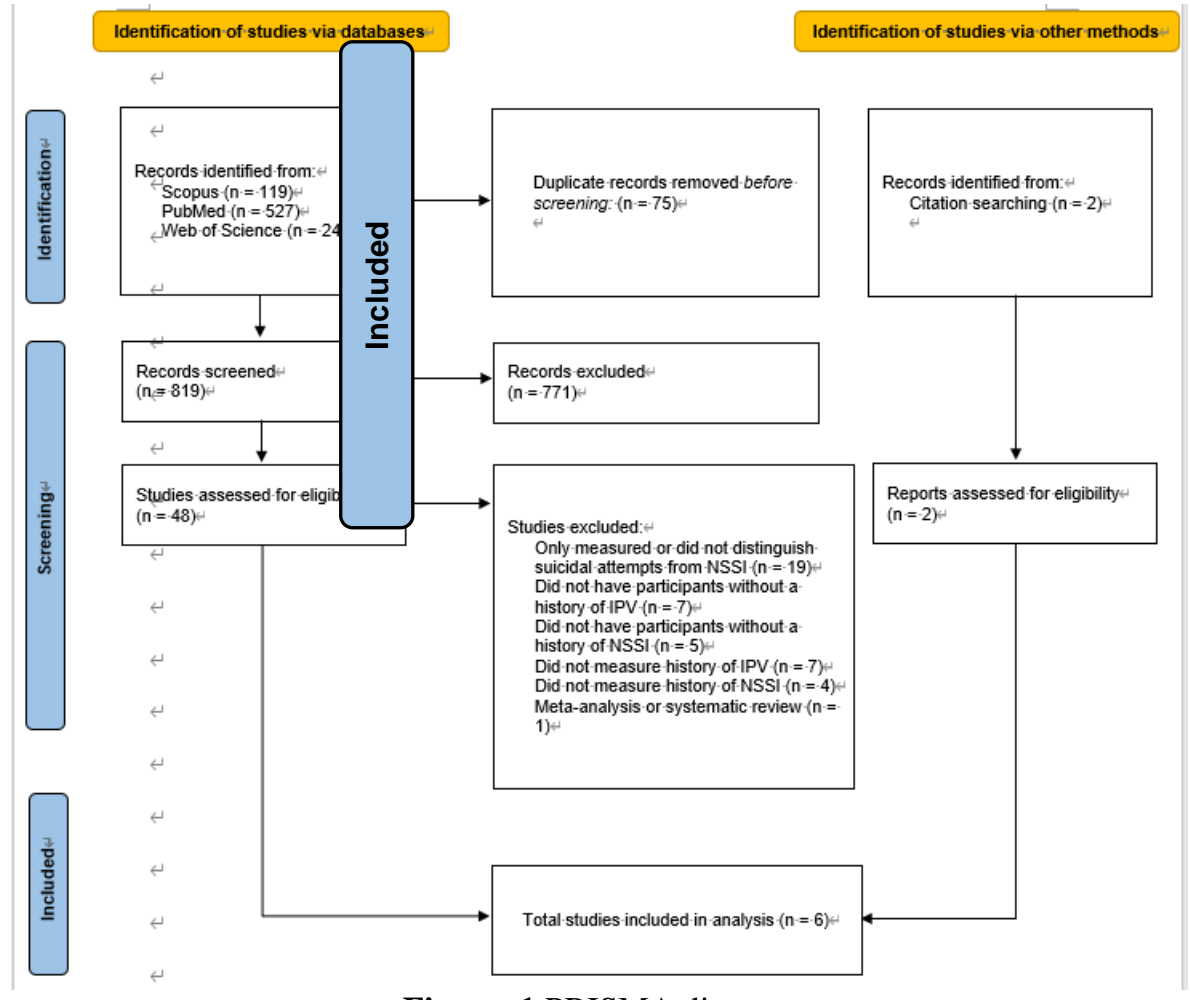

Figure. 1 PRISMA diagram 
The search strategy yielded 894 studies initially. After excluding 75 duplicate studies, 819 studies were screened. Among these, 771 were excluded (Fig. 1). The remaining 48 studies were further examined based on inclusion and exclusion criteria. However, 19 of them did not distinguish non-suicidal self-injury from suicidal attempts. Another 12 studies were excluded either because they did not include participants without exposure to intimate partner violence or because they did not include participants without a history of non-suicidal self-injury. Moreover, for one of the remaining studies there was not enough information to extract an odds ratio and the effort to request the data from the authors was unsuccessful. Overall, only 6 studies with known odds ratios were included in the meta-analysis. Reference sections of the included studies were also inspected to ensure no additional relevant study was missed by the initial search strategy. Only two additional studies were located, and both did not include participants without a history of non-suicidal self-injury. Therefore, it could be concluded that that the initial search strategy was sufficiently comprehensive and inclusive. A total of 6 studies met full inclusion criteria and were extracted for the meta-analysis. These 6 studies provided odds ratios from 8 independent samples.

\subsection{Data analysis}

For each study in the meta-analysis, odds ratios indicating the association between exposure to intimate partner violence and non-suicidal self-injury were extracted or converted from the reported prevalence of non-suicidal self-injury in the exposure group and the control group. An odds ratio is a measure of association between an exposure and an outcome. In this case, it represented the odds that non-suicidal self-injury would occur given the exposure to intimate partner violence, compared to the odds of non-suicidal self-injury occurring without exposure to intimate partner violence. The final analysis was conducted using OpenMeta[Analyst] from Brown University ${ }^{[16]}$. A random-effects model was used since the included studies were not functionally equal.

A summary of study details and extracted data can be found in Table 1. Out of the 6 studies included, 4 studies used college student samples while the other two sampled only transgender individuals. Most studies used young samples, with mean ages of participants being around 25 years or younger. Additionally, most studies had more female participants than male participants, except in which the biological sex of the sampled transgender individuals was not clearly stated ${ }^{[17]}$.

\subsection{Results}

Table 1 gives an overview of the key characteristics and relevant outcomes from each included study. A total of 6 studies and 8 independent samples reported a comparison between the likelihood of developing NSSI in IPV and control groups. All but one of these studies indicated that exposure to IPV predicted a higher likelihood of NSSI behaviors ${ }^{[17]}$.

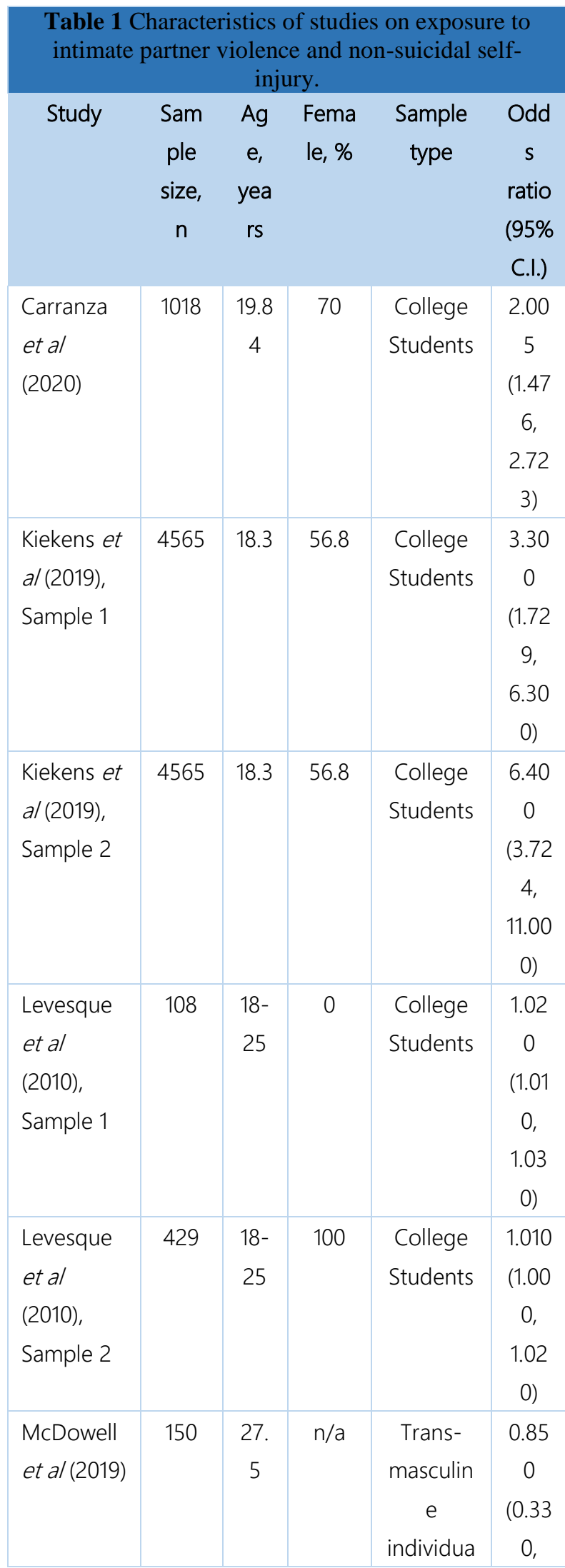




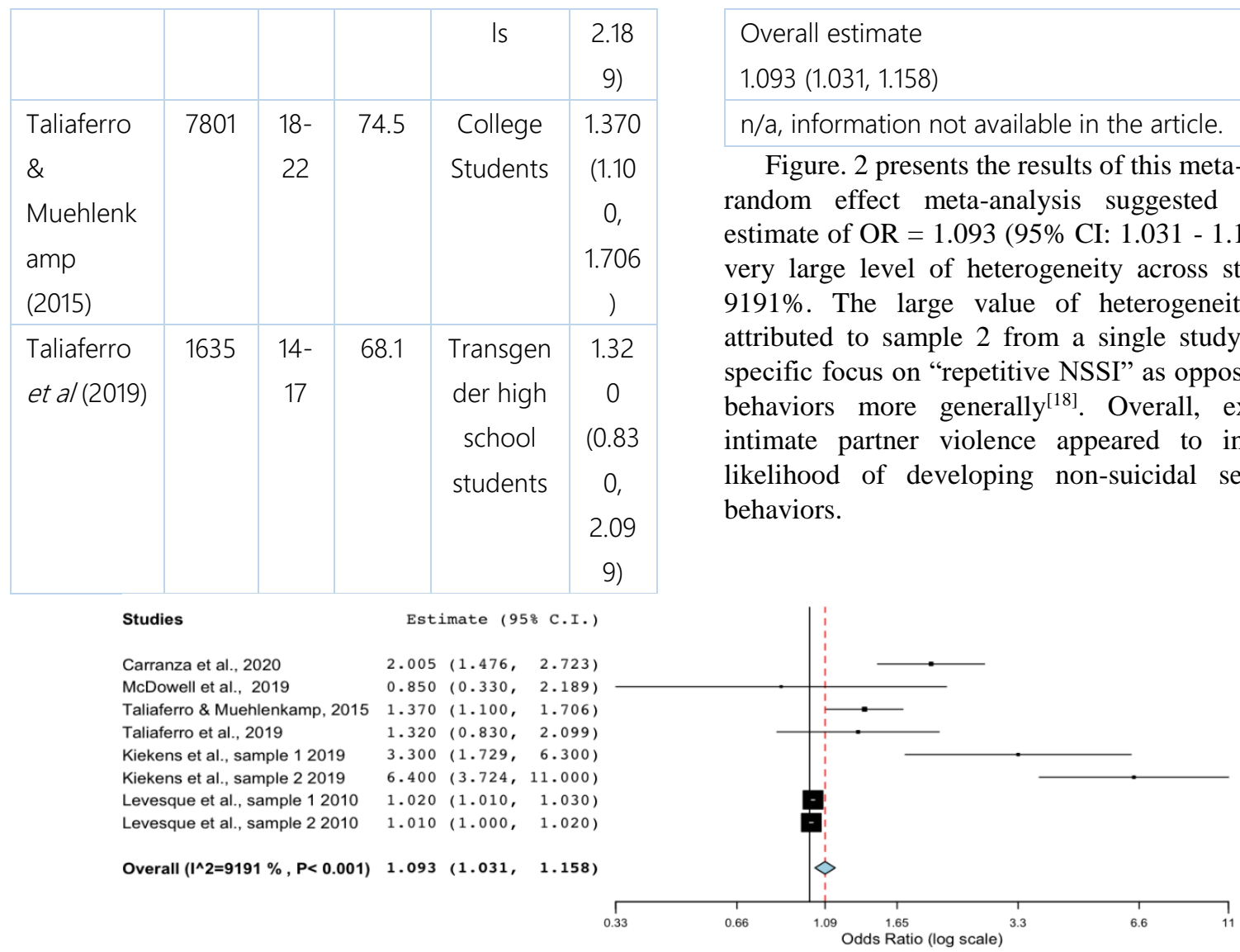

Figure. 2 Forest plot of meta-analysis on the likelihood of NSSI behaviors with or without previous exposure to intimate partner violence.

\section{CONCLUSION}

This meta-analysis examined the association between exposure to intimate partner violence and the development of non-suicidal self-injurious behaviors. Across the 8 samples from 6 included studies, the overall OR was $1.093(95 \% \mathrm{CI}=1.031-1.158)$, indicating greater odds of developing non-suicidal self-injury given exposure to intimate partner violence. This result suggests individuals with exposure to intimate partner violence will be more at risk for developing non-suicidal self-injurious behaviors. However, the value also indicates a relatively weak relationship between the two. Therefore, it is unlikely that intimate partner violence has a primary role in the development of non-suicidal selfinjury. This finding targeted specific population at risk for developing NSSI and offered insights about interpreting the etiology of non-suicidal self-injury.

In addition, the effect intimate partner violence had on non-suicidal self-injury was less pronounced in transgender individuals. Studies which sampled transgender individuals tended to report lower odds ratios compared with studies that mainly focused on cisgender individuals. In fact, one of the included studies which sampled 150 female-to-male trans-masculine individuals reported OR $=0.850(95 \% \mathrm{CI}: 0.330-$
2.189), implying lower odds of developing non-suicidal self-injury given experience with intimate partner violence $^{[17]}$. However, it should be acknowledged that this study had a much smaller sample size compared with the rest. Also, it is expected that these individuals could have faced unique circumstances and challenges in their intimate relationships compared with cisgender individuals. Moreover, most of the included studies had more females than males in their samples. This suggests the possibility of a bias as gender could be a confounding factor mediating the relationship between intimate partner violence and non-suicidal self-injury. Previous literature has suggested that gender moderates the relationship between the nature of intimate partner violence and help-seeking behaviors ${ }^{[19,20]}$. Compared with men, women are more likely to seek help for intimate partner violence victimization and thus less likely to develop self-injurious behaviors to alleviate their negative emotions ${ }^{[19]}$. Given such finding, it was possible that a stronger relationship would be found between intimate partner violence and non-suicidal selfinjury if gender was controlled.

Last but not the least, the findings from included studies were largely related to Caucasian, Western samples. Therefore, it remains unclear whether such association also exists in other ethnic or cultural groups. Future studies should try to incorporate individuals from 
more diverse backgrounds to provide a more wholistic view on consequences following exposure to intimate partner violence. Similarly, since most of the included studies only measured non-suicidal self-injury within an individual's lifetime, it is hard to differentiate between development and maintenance of such behavior. In the current meta-analysis, only one included study differentiated between sporadic and repetitive nonsuicidal self-injury ${ }^{[18]}$. Noticeably, a much higher odds ratio between intimate partner violence and non-suicidal self-injury was found among individuals who engaged in non-suicidal self-injury repetitively, indicating a possible role of intimate partner violence exposure for maintaining self-injurious behaviors. Therefore, future studies should also aim to investigate this potential association by differentiating individuals who engage in non-suicidal self-injury repetitively from those whose engagements are less persistent. Clinical practitioners and social workers will likely benefit from such finding to provide treatment or help tailored for different individuals' unique circumstances to help combat the problem of non-suicidal injury.

\section{REFERENCES}

[1] Nock, M. K., \& Favazza, A. R. (2009). Nonsuicidal self-injury: Definition and classification. In: M.K. Nock (Ed.), Understanding nonsuicidal self-injury: Origins, assessment, and treatment. American Psychological Association, Washington. pp. 9-18.

[2] Zetterqvist, M. (2015). The DSM-5 diagnosis of nonsuicidal self-injury disorder: A review of the empirical literature. Child and Adolescent Psychiatry and Mental Health, 9: 31.

[3] Cipriano, A., Cella, S., \& Cotrufo, P. (2017). Nonsuicidal Self-injury: A Systematic Review. Frontiers in Psychology, 8: 1946.

[4] Langbehn, D. R., \& Pfohl, B. (1993). Clinical correlates of self-mutilation among psychiatric inpatients. Annals of Clinical Psychiatry: Official Journal of the American Academy of Clinical Psychiatrists, 5(1): 45-51.

[5] Tantam, D., \& Whittaker, J. (1992). Personality disorder and self-wounding. The British Journal of Psychiatry: The Journal of Mental Science, 161: 451-464.

[6] Yates, T. M. (2004). The developmental psychopathology of self-injurious behavior: Compensatory regulation in posttraumatic adaptation. Clinical Psychology Review, 24(1): 3574.

[7] Campbell, J. C. (2002). Health consequences of intimate partner violence. Lancet, 359(9314): 13311336.
[8] Dutton, M. A., Green, B. L., Kaltman, S. I., Roesch, D. M., Zeffiro, T. A., \& Krause, E. D. (2006). Intimate partner violence, PTSD, and adverse health outcomes. Journal of Interpersonal Violence, 21(7): 955-968.

[9] Ansara, D. L., \& Hindin, M. J. (2010). Formal and informal help-seeking associated with women's and men's experiences of intimate partner violence in Canada. Social Science \& Medicine (1982), 70(7): 1011-1018.

[10] Beydoun, H. A., Beydoun, M. A., Kaufman, J. S., Lo, B., \& Zonderman, A. B. (2012). Intimate partner violence against adult women and its association with major depressive disorder, depressive symptoms and postpartum depression: A systematic review and meta-analysis. Social Science \& Medicine (1982), 75(6): 959-975.

[11] Bonomi, A. E., Anderson, M. L., Reid, R. J., Rivara, F. P., Carrell, D., \& Thompson, R. S. (2009). Medical and psychosocial diagnoses in women with a history of intimate partner violence. Archives of Internal Medicine, 169(18): 1692-1697.

[12] Devries, K., Watts, C., Yoshihama, M., Kiss, L., Schraiber, L. B., Deyessa, N., Heise, L., Durand, J., Mbwambo, J., Jansen, H., Berhane, Y., Ellsberg, M., Garcia-Moreno, C., \& WHO Multi-Country Study Team. (2011). Violence against women is strongly associated with suicide attempts: Evidence from the WHO multi-country study on women's health and domestic violence against women. Social Science \& Medicine (1982), 73(1): 79-86.

[13] Klonsky, E. D., \& Muehlenkamp, J. J. (2007). Selfinjury: a research review for practitioner. Journal of Clinical Psychology, 63(11):1045-1056.

[14] Sansone, R. A., Chu, J., \& Wiederman, M. W. (2007). Self-inflicted bodily harm among victims of intimate-partner violence. Clinical Psychology \& Psychotherapy, 14(5): 352-357.

[15] Vaughn, M. G., Salas-Wright, C. P., DeLisi, M., \& Larson, M. (2015). Deliberate self-harm and the nexus of violence, victimization, and mental health problems in the United States. Psychiatry Research, 225(3): 588-595.

[16] Wallace, B., Trikalinos, T., Lau, J., Trow, P., \& Schmid, C. (2011). Closing the Gap between Methodologists and End-Users: $\mathrm{R}$ as a Computational Back-End. Journal of Statistical Software, 49(5): 1-15.

[17] McDowell, M. J., Hughto, J. M. W., \& Reisner, S. L. (2019). Risk and protective factors for mental health morbidity in a community sample of female- 
to-male trans-masculine adults. BMC Psychiatry, 19(1): 16 .

[18] Kiekens, G., Hasking, P., Claes, L., Boyes, M., Mortier, P., Auerbach, R. P., Cuijpers, P., Demyttenaere, K., Green, J. G., Kessler, R. C., Myin-Germeys, I., Nock, M. K., \& Bruffaerts, R. (2019). Predicting the incidence of non-suicidal self-injury in college students. European Psychiatry: The Journal of the Association of European Psychiatrists, 59: 44-51.

[19] Cho, H., Seon, J., Han, J.-B., Shamrova, D., \& Kwon, I. (2020). Gender Differences in the Relationship Between the Nature of Intimate Partner Violence and the Survivor's Help-Seeking. Violence Against Women, 26(6-7): 712-729.

[20] Monterrosa, A. E. (2019). How Race and Gender Stereotypes Influence Help-Seeking for Intimate Partner Violence. Journal of Interpersonal Violence, 36(17-18): NP9153-NP9174. 